INPLASY

PROTOCOL

To cite: Visram et al.

Comparing visual

reinforcement audiometry

outcomes using different

auditory stimuli and visual

rewards. Inplasy protocol

202110080. doi:

10.37766/inplasy2021.1.0080

Received: 20 January 2021

Published: 20 January 2021

Corresponding author:

Anisa Visram

anisa.visram@manchester.ac.uk

Author Affiliation:

University of Manchester

Support: William Demant

Foundation.

Review Stage at time of this submission: Preliminary searches.

Conflicts of interest:

None to declare.

\section{Comparing visual reinforcement audiometry outcomes using different auditory stimuli and visual rewards}

Visram, AS1; Jackson, IR2; Almufarrij, I3; Stone, MA4; Munro, KJ5.

Review question / Objective: This mapping review aims to map out different types of acoustic stimuli and visual rewards used in VRA, and to explore how different acoustic stimuli or visual rewards can improve VRA outcomes, with a focus on infants, but not excluding older children and adults in whom VRA is a developmentally appropriate assessment. VRA outcomes may include the number of MRLs obtained, the number of reliable responses obtained, time taken to complete testing, or other measures of how successful the test was. The focus of the mapping review will be on different auditory stimuli and rewards used in the procedure and how these affect test outcome. Should the search uncover other approaches that impact on success of the test, these will also be reported.

Condition being studied: Performance in infant behavioural hearing testing (Visual Reinforcement Audiometry).

INPLASY registration number: This protocol was registered with the International Platform of Registered Systematic Review and Meta-Analysis Protocols (INPLASY) on 20 January 2021 and was last updated on 20 January 2021 (registration number INPLASY202110080).

\section{INTRODUCTION}

Review question / Objective: This mapping review aims to map out different types of acoustic stimuli and visual rewards used in VRA, and to explore how different acoustic stimuli or visual rewards can improve VRA outcomes, with a focus on infants, but not excluding older children and adults in whom VRA is a developmentally appropriate assessment. VRA outcomes may include the number of MRLs obtained, the number of reliable responses obtained, time taken to complete testing, or other measures of how successful the test was. The focus of the mapping review will be on different auditory stimuli and rewards used in the procedure and how these affect test 
outcome. Should the search uncover other approaches that impact on success of the test, these will also be reported.

Rationale: This information will help clinicians to better understand the relative benefits of different approaches to the technique, and may highlight areas for further research.

Condition being studied: Performance in infant behavioural hearing testing (Visual Reinforcement Audiometry).

\section{METHODS}

Search strategy: The following search strategy was used for PubMed, and adapted as needed for different databases: ("visual reinforce*"[Text Word] OR "visually reinforce*"[Text Word] OR "conditioned orienta*"[Text Word] OR "conditioned head-turn"[Text Word] OR "operant audiometry"[Text Word] OR "visual reward*"[Text Word] OR "conditioned visual"[Text Word]) AND ("audiometr*"[Text Word] OR "Audiometry"[Mesh] OR "reflex*"[Text Word] OR "auditory"[Text Word] OR "discrimination"[Text Word] OR "fixation"[Text Word]).

Participant or population: Primarily infants, but not excluding older children and adults in whom Visual Reinforcement Audiometry is a developmentally appropriate assessment.

Intervention: Different stimuli and approaches used in Visual Reinforcement Audiometry.

Comparator: With or without any comparator.

Study designs to be included: There will be no limitation on the design of the included studies.

Eligibility criteria: This review will include any published studies that report on approaches to VRA testing. This may include use of different auditory stimuli, visual rewards, presentation times, or any other investigations that aim to improve the outcomes of the test. The outcome of the test may be any metric relating to the success of the test, including but not limited to the number of reliable responses shown by the participant, the number of MRLs obtained for the participant, proportion of infants reaching a test completion criterion, or time/number of visits needed to reach completion criterion. There will be no limitation on the design of the included studies. There will be no restriction on participant age, hearing status, or cognitive ability. Non-English language papers will be considered where an English abstract is provided. The reference lists of relevant published clinical guidelines, including the British Society of Audiology recommended procedure for visual reinforcement audiometry (BSA, 2014) will be reviewed for additional studies to include. Grey literature, including magazine articles, will also be included. Preprints, clinical guidelines and book chapters will be excluded.

Information sources: PubMed, PsycINFO, EMBASE, EMCare, Cochrane Library, Global Health, Academic Search Premier, Web of Science, Google Scholar.

Main outcome(s): Outcomes may include the number of Minimum Response Levels obtained, the number of reliable responses obtained, time taken to complete testing, or other measures of how successful the test was.

Data management: Records retrieved by the search will be exported to reference management software for automated removal of duplicates. A further, manual assessment will be then be performed to identify and remove any remaining duplicates not detected in the automated search. Data from the identified studies will be summarised in a spreadsheet which will include: publication details (title, author(s), publication year, journal, contact details), study design, rewards used, auditory stimuli, participant demographics, outcomes, funding, and any other relevant data. 
Quality assessment / Risk of bias analysis: As a mapping review exercise there will be no formal quality assessment of the studies.

Strategy of data synthesis: Data will be combined and reported narratively.

Subgroup analysis: As a mapping exercise, there is no plan to analyse data in subgroups.

Sensibility analysis: As a mapping exercise, there is no plan to perform sensibility analysis.

Language: No restrictions will be placed on language.

Country(ies) involved: United Kingdom.

Keywords: visual reinforcement audiometry; infant; hearing; behavioural.

Contributions of each author:

Author 1 - Anisa Visram - The author drafted the protocol.

Email: anisa.visram@manchester.ac.uk

Author 2 - lain Jackson - The author revised successive versions of the protocol.

Email: iain.jackson@manchester.ac.uk

Author 3 - Ibrahim Almufarrij - The author revised successive versions of the protocol.

Email: ibrahim.almufarrij@manchester.ac.uk Author 4 - Michael Stone - The author revised successive versions of the protocol.

Email: michael.stone@manchester.ac.uk Author 5 - Kevin Munro - The author revised successive versions of the protocol.

Email: kevin.j.munro@manchester.ac.uk 